\title{
Sudden transitions and grand variations in the solar dynamo, past and future
}

\author{
Cornelis De Jager ${ }^{1, *}$ and Silvia Duhau ${ }^{2}$ \\ 1 Royal Netherlands Institute for Sea Research, PO Box 59, 1790 AB Den Burg, The Netherlands \\ *corresponding author: e-mail: info@cdejager.com \\ 2 Departamento de Física, Facultad de Ingenieria, Universidad de Buenos Aires, 1428 Buenos Aires, Argentina.
}

Received 21 February 2012 / Accepted 11 June 2012

\begin{abstract}
The solar dynamo is the exotic dance of the sun's two major magnetic field components, the poloidal and the toroidal, interacting in anti-phase. On the basis of new data on the geomagnetic $a a$ index, we improve our previous forecast of the properties of the current Schwabe cycle \#24. Its maximum will occur in 2013.5 and the maximum sunspot number $R_{\max }$ will then be $62 \pm 12$, which is within the bounds of our earlier forecasts. The subsequent analysis, based on a phase diagram, which is a diagram showing the relation between maximum sunspot numbers and minimum geomagnetic $a a$ index values leads to the conclusion that a new Grand Episode in solar activity has started in 2008. From the study of the natural oscillations in the sunspot number time series, as found by an analysis based on suitable wavelet base functions, we predict that this Grand Episode will be of the Regular Oscillations type, which is the kind of oscillations that also occurred between 1724 and 1924. Previous expectations of a Grand (Maunder-type) Minimum of solar activity cannot be supported. We stress the significance of the Hallstatt periodicity for determining the character of the forthcoming Grand Episodes. No Grand Minimum is expected to occur during the millennium that has just started.
\end{abstract}

Key words. Sun - dynamo - active regions - toroidal field - poloidal field

\section{The dynamo}

The solar dynamo is the mechanism that runs solar variability. Its essential part is the tachocline, a layer with a vertical extension of about $20000 \mathrm{~km}$, near the base of the outer solar convection region, about $200000 \mathrm{~km}$ below the surface (Hujeirat \& Yorke 1998; Brun et al. 2002; Cally et al. 2003). At that level the interplay of convective motions and differential solar rotation leads to the origin of a complicated and to some extent chaotic system of magnetic fields and associated electric currents (Charbonneau et al. 1999; Basu \& Schou 2000). Due to the internal solar shearing motions, the magnetic fields are stretched into a direction parallel to the solar equator. Thus, it becomes a field system that is mainly toroidal. By continued stretching the toroidal field strength increases in the course of subsequent solar rotations. When these field strengths reach values of the order of $10^{5}$ Gauss (several determinations, reviewed by De Jager 2005, yield an average value of $\sim 80 \mathrm{kG}$ ), this toroidal field becomes unstable. Smaller closed loops detach from it, due to kink instability. They have smaller specific weights and hence stronger buoyance than the surrounding solar gas, because of their high magnetic field strengths. Therefore, these loops rise, to reach the solar photosphere after one or a few months. There they appear as a pair of sunspots (Solanki et al. 2006).

Later in the life of the toroidal field, it further disintegrates and weaker loops will also detach. They rise slower because their magnetic buoyance is only marginally larger than that of the surroundings. Because of the Coriolis force the rising trajectory is roughly parallel to the solar axis. Underway they rotate

\footnotetext{
The full text of all quoted papers by the two authors of this paper can be consulted at www.cdejager.com/sun-earth-publications/.
}

slightly $\left(\sim 90^{\circ}\right)$, due to the Coriolis force and thus they arrive as a poloidal field in the polar areas of the sun. Hence, the polar field component has mainly a poloidal character.

This is a concise, necessarily schematic description of the solar dynamo. It is briefly characterized by the Omega effect, which is the magnetic field strengthening in a direction parallel to the equator, leading to a field component that is mainly toroidal with a shape that somewhat resembles the Greek capital Omega (cf., e.g., Cally et al. 2003), and the Alpha effect which is the formation of the poloidal field under influence of the Coriolis force leading to alpha-type field loops (Küker et al. 2001). Extended descriptions and analyses are found in many review papers (we refer to the reviews by Fisher et al. 2000; Tobias 2002; Ossendrijver 2003; Charbonneau 2010; De Jager \& Duhau 2011).

Hence, the dynamo consists essentially of a toroidal and a poloidal field component, that are out of phase in so far as the integrated values of their field strengths are considered. When the dipolar part of the poloidal field has acquired maximal strength, the toroidal field is at minimum and reversely so (Fig. 1). The period of variation is around 22 years, the Hale cycle. Half of it, an $\sim 11$-year period is called the Schwabe cycle. When during one Schwabe cycle the toroidal field component would be directed east-west, it will be directed oppositely during the subsequent and previous Schwabe cycles. A comparable situation applies to the poloidal field: When it would be polarized north-south at minimum of a toroidal ("sunspot", "Schwabe") cycle, it will be directed the opposite way at the subsequent minimum (Fig. 1). For this reason the Hale cycle is more fundamental than the Schwabe cycle.

At this point, again referring to Figure 1, we signalize the deviating behaviour of the two curves in the years from 2006 


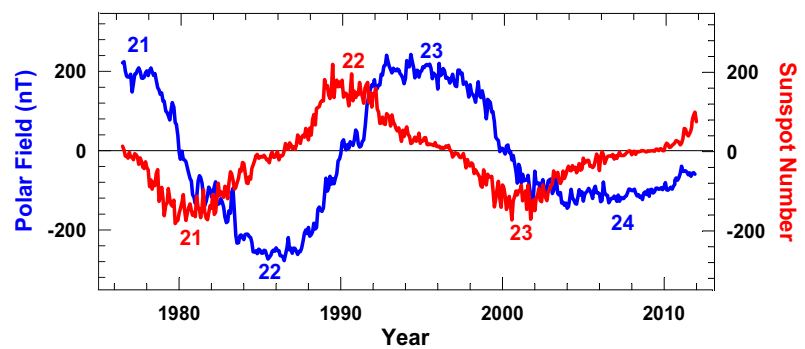

Fig. 1. The Hale magnetic cycle in the north-south average of the polar field, as observed at the Wilcox Solar Observatory (http:// wso.stanford.edu) in the interval 1975.5-2010 (blue) and the sunspot number curve (red) to which the sign is assigned according to the toroidal field polarity of successive Schwabe cycles. The polar maxima are numbered by the standard numbers of the next following sunspot maxima.

onwards, a surprising phenomenon. We consider this an indication of an ongoing fundamental change in solar activity. Later in this paper (Sects. 4 and 5) we return to that aspect.

\section{Proxies for the dipolar and the toroidal magnetic field strengths}

Since direct measurements of the magnetic field strengths at the solar surface are available for limited time intervals only (the equatorial fields since the beginning of the 20th century; the polar since around 1976), it is useful to use "proxies" for extending the intervals.

A proxy for the amplitude of the Schwabe cycle in the toroidal field component, henceforth called the "strength" of the toroidal field, is the value of sunspot number $R$ at sunspot maximum: $R_{\max }$. Fairly complete data for Sunspot numbers are known since 1610 . The specific rationale for assuming $R$ as a proxy for the toroidal field strength is the following. At solar maximum the poloidal component of the magnetic field passes through zero and hence the magnetic flux at the equator is entirely due to the toroidal field. The total sunspot area $(S$; Nagovitsyn 2005) is proportional to the total magnetic flux of the spots. By scaling the $R$ values to the $S$ scale we derived the empirical relation $S\left(M_{\mathrm{sh}}\right)=15.7 R$, where $M_{\mathrm{sh}}$ is the spot area expressed in millionths of the solar hemisphere. It is only in the period 1704-1780 that there are some minor deviations from this empirically derived rule. Hence, we may conclude that the $R_{\max }$ values are proportional to the maximum strengths of the related toroidal fields.

The foregoing discussion allows us to use $R_{\max }$ as a reliable proxy for the strength of the toroidal field component.

We next discuss the proxy for the poloidal field. Russell (1975; cf. also Russell \& Mulligan 1955) found a long-term rise in geomagnetic activity and proposed that this is caused by changes in the sun's polar magnetic field strength. This longterm rise seems to be due to an increase in the background solar wind disturbances during sunspot minima, rather than to the number density of solar wind disturbances (Vennerstroem 2000), a fact that supports Russell's suggestion. Geomagnetic index minima occur around sunspot minima, when the toroidal field component passes through zero (blue line in Fig. 1) and solar wind disturbances assume minimum values. At that time the poloidal field is mainly dipolar. The value of the geomagnetic index during the minima of subsequent Schwabe cycles has been proposed to be a measure for the amplitude of the

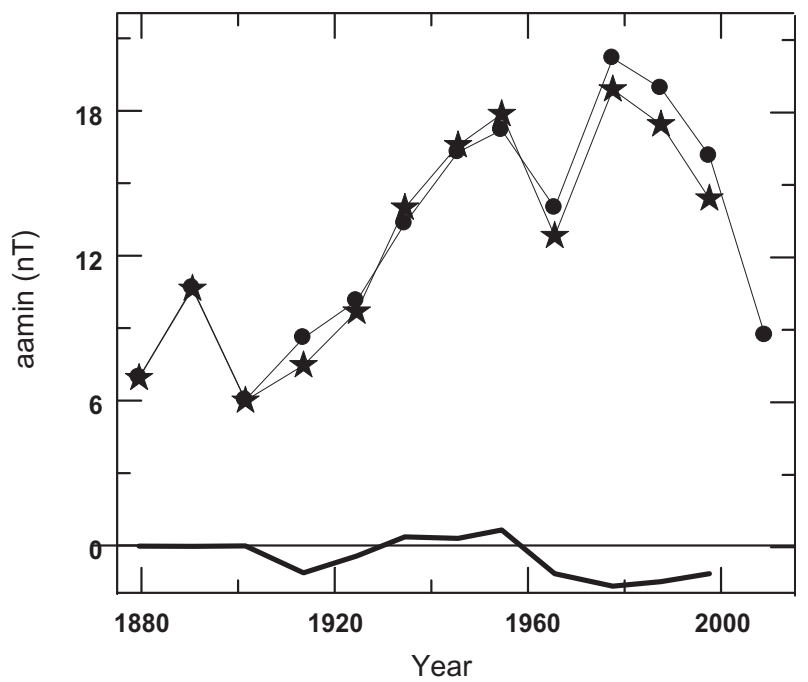

Fig. 2. A comparison of the time series of the Standard $a a$ data ((ftp://.ngfc.noaa.gov/STP/SOLARDATA/REALTED_INDICES/ AA_INDEX/AA_YEAR) (points) and the Lockwood data (stars; priv. comm., 2009). The line at the bottom of the figure is the difference between the two time series.

Schwabe cycle in the dipolar field, hence of the dipolar field strength, $D M_{\max }$ (Schatten et al. 1978; Layden et al. 1991; Legrand \& Simon 1991; Hathaway et al. 1999; Duhau \& Chen 2002).

The geomagnetic index for which the longest time series is available is the $a a$ index introduced by Mayaud (1975). The validity of any analysis based on $a a$ is based on the assumed constancy of the calibration of this index over time. Svalgaard et al. (2004) have presented evidence of the non-constancy of the $a a$ calibration. Lockwood et al. (2006) employed data from a variety of geomagnetic monitoring stations to evaluate the accuracy of the long-term drift observed in the $a a$ geomagnetic index and found consistent evidence that the geomagnetic index $a a$ appeared to be systematically slightly lower (by about $2 \mathrm{nT}$ ) during 1868-1957, as compared with its usually reported "standard" values (cf. Fig. 2). Much of the discrepancy appears to arise from the move of the northern hemisphere geomagnetic station from Abinger to Hartland in 1957. The "standard" value of $a a$ during sunspot minimum, $a a_{\text {min }}$ is currently used in most applications, but in view of the presently ongoing discussion on the accuracy of the geomagnetic index aa during the last century we will consider, throughout this paper, these two different versions of this geomagnetic index. Henceforth, we will call them the standard and the Lockwood values (Fig. 2).

It appears that there exists a fine linear relationship between the $a a_{\min }$ values and the observed solar polar field strength at its maximum $D M_{\max }$, as was published for the first time by De Jager \& Duhau (2009; cf. their Fig. 3). A view on the longterm trend (see the present Fig. 2) of the difference between the two versions of the geomagnetic index during the last three sunspot cycles (the only cycles during which the polar fields have been measured systematically) leads to a larger slope in the regression lines when computed from the standard data as compared with those based on Lockwood's data (Fig. 3).

There is empirical evidence that the strength of a given Schwabe cycle in the toroidal field is related to the strength of the preceding cycle in the dipolar field (Ohl 1966; Callebaut, priv. comm.). For reviews on these various aspects of the dynamo (cf. Hathaway et al. 1999; Duhau \& Chen 2002; 
C. De Jager and S. Duhau: Sudden transitions and grand variations

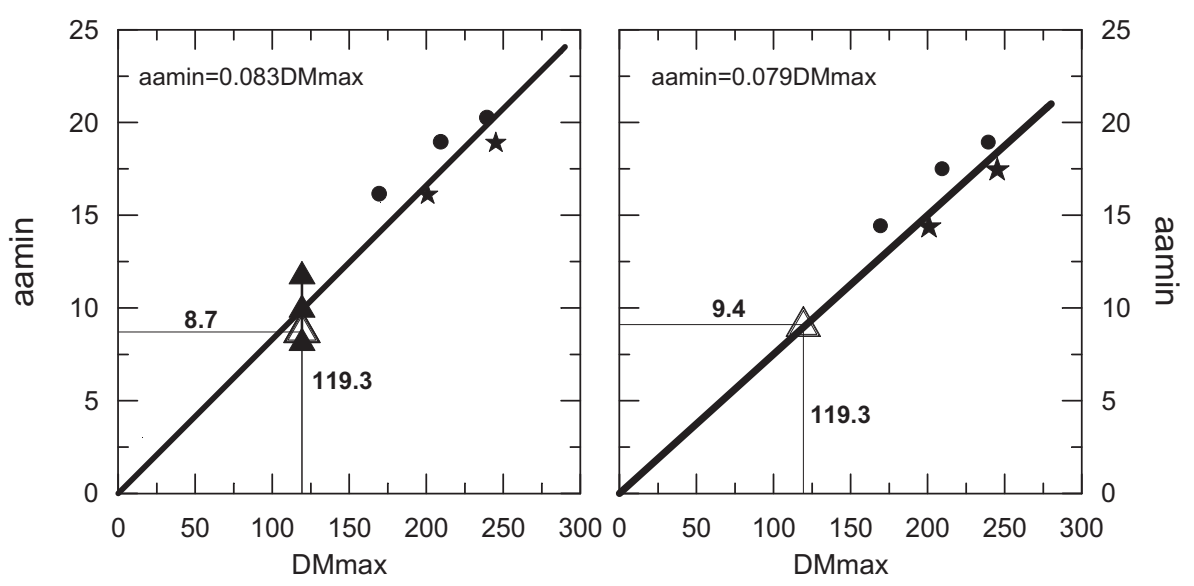

Fig. 3. Geomagnetic index at minimum, $a a_{\min }$, vs. observed maximum amplitude of the dipolar field strength, $D M_{\max }$. The ordinates of the stars and dots are the observations of $D M_{\max }$ as deduced from Mount Wilson and Wilcox solar observatories data, respectively. The abscissae are the yearly values of the standard $a a$ index (left-hand panel) and the homogenized data from Lockwood (2009, priv. comm.). The filled triangles in the left-hand panel are a measure for the prediction of $a a_{\min } \# 24$ as derived from the regression line and the value of $D M_{\max }$ for cycle 24 (De Jager \& Duhau 2009) and the open triangle is the observed value. The open triangle in the right-hand panel is the value of $a a_{\text {min }}$ obtained from the corresponding regression line. This diagram proves that $a a_{\min }$ is a suitable proxy for the polar field flux.

Duhau \& De Jager 2008). Even with the relative small quantitative difference between the two versions of the geomagnetic index $a a$ (thick line at the bottom of Fig. 2), it does show a long-term component during the past 20th century Grand Maximum. As a result the two time series lead to similar short-term predictions but also to a substantial qualitative difference in the long-term prediction, as we discuss below in Sections 3 and 5.

\section{Grand Episodes and the Transition Point; the Phase Diagram; some remarks on the long-term prediction of solar activity}

A plot of the sunspot number $R$ against time (Fig. 4) shows the succession of Schwabe cycles. It is also very apparent that the strengths of the various cycles do vary considerably.

Another aspect is that there is some system in these apparently chaotic variations. There are three kinds of Grand Episodes (Duhau \& De Jager 2010; De Jager \& Duhau 2009, 2011), those of high solar activity, such as the Grand Maximum of the 20th century, and in contrast we know a period of extremely low activity, the Grand (Maunder) Minimum of the 17th century. In between these two Grand Episodes was a period of fairly Regular Oscillations, during which a short and shallow minimum occurred, the Dalton Minimum ( 1790 to $\sim 1830)$. In this section we will show that in 2008 a transition to another Grand Episode took place. Its character will be discussed in Sections 4 and 5.

Solar long-term variability is well shown in a phase diagram (Fig. 5). The phase diagram was introduced by us (Duhau $\&$ De Jager 2008, 2010). It shows the relation between $R_{\max }$ for the subsequent Schwabe cycles and the value of the preceding $a a_{\text {min }}$ value. In other words, it shows the relation between the two proxies for the amplitudes of the Schwabe cycle in the two main solar field components in the course of time. The diagram is obtained after having subtracted all wavelet components with a Fourier period below 17 years. Apart from that it contains all the oscillations in the times series.

We have found that a transition between Grand Episodes occurs when these two proxies (Duhau \& De Jager 2008,
2010), those of the toroidal and the poloidal fields, assume simultaneously two well-defined values. A so-called Grand Transition will occur when at the same time $R_{\max }=94.4$ (sunspot numbers) and $a a_{\min }=10.3 \mathrm{nT}$ (in the standard time series). These two parameters define the Transition Point. The $a a_{\min }-$ $R_{\text {max }}$ curve passed through the Transition Point in 1620 - the onset of the Maunder Minimum; again in 1924 - onset of the modern (20th century) Grand Maximum. During the return to the Transition Point after the Maunder Minimum, in 1724, the path appeared to slightly miss the Transition Point where after an episode of Regular Oscillations started. That happened too during previous transitions to Regular Oscillations (Duhau \& De Jager 2008). Whether this is a universal rule is difficult to state; lack of sufficiently accurate secular data hampers a generalization of this assumption.

Figure 5 also shows that the recent Grand Maximum lasted from 1924 till 2008. In that latter year the curve did not pass exactly through the Transition Point in the left-hand diagram. It did pass through it in the other diagram. This ambivalence makes it impossible to decide at this point whether or not we are at present witnessing the start of another episode of Regular Oscillations as seems to be suggested by the left-hand diagram or whether we experience the start of a Grand Minimum (righthand diagram). This question will next be discussed and we return to it in Section 5.

By applying a suitable wavelet representation we (review by De Jager \& Duhau 2010) found that solar long-term variability is determined by three significant components: (Fig. 6), viz. the bi-decadal, semi-secular and secular oscillations. The latter is similar but not identical to the Gleissberg cycle. The secular cycle as defined here does not contain wavelet components with Fourier periods comprised within the lower Gleissberg band. These, instead, are included in the semi-secular oscillations. The rationale for this choice is that the lower and the upper Gleissberg bands of periods appear to arise from different phenomena in the Sun (cf. Duhau \& De Jager 2010). The Grand Episodes are due to a sudden change of the length and amplitude of the secular oscillation, but that statement does not apply to the Dalton Minimum. The latter, instead, is due to a strong semi-secular oscillation of which the relative minimum occurs 


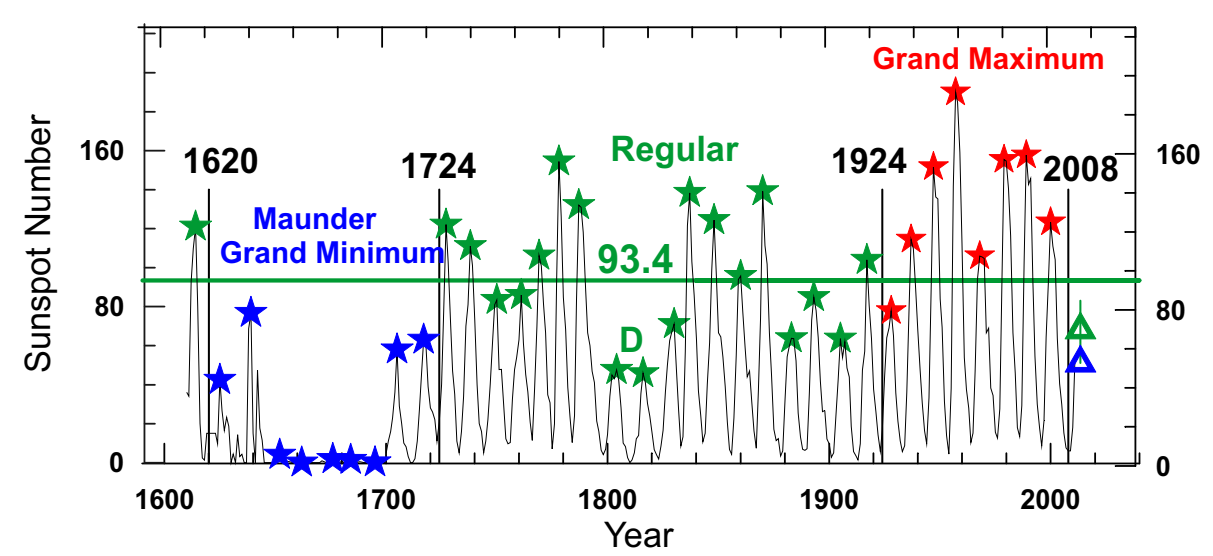

Fig. 4. The sunspot cycles since 1610. The diagram shows the sunspot numbers plotted against time. The stars are the maximum values of the sunspot (Schwabe) cycles. The strong variations are apparent. The three Grand Episodes are marked by their different colours. They are separated by vertical black lines - cf. Figure 5, later this section. The open triangles are our predictions, based on the two types of $a a$ data used, viz. the standard data (green; De Jager \& Duhau 2009) and the Lockwood data (blue; Duhau \& De Jager 2010).The vertical green line through the green triangle is the estimated error. The horizontal line at sunspot number $R \approx 94$ marks the level of the Transition Point, cf. its definition later in this section.
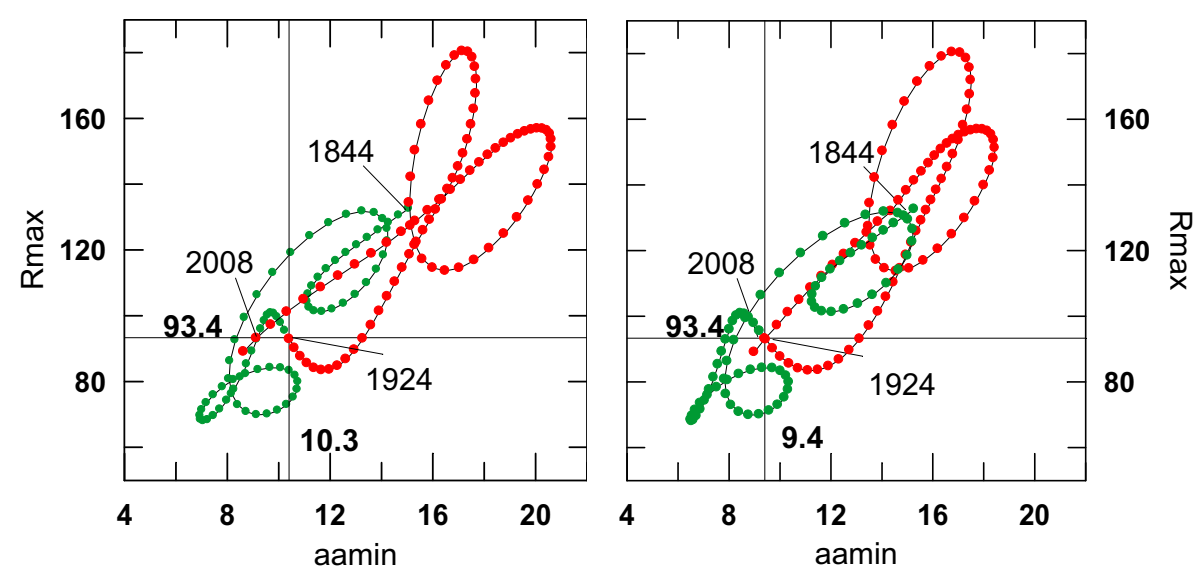

Fig. 5. The phase diagram gives one point per year for the period 1844-2011. The left-hand diagram is for the standard data; the other is for the Lockwood data. Data prior to 1968 are from Nevanlinna \& Kataja (1993) in the two panels. From 1968 onward the $a a_{\text {min }}$ data are as given in Figure 2. The abscissa and ordinate of the horizontal and vertical lines are the coordinates of the Transition Point. There is a difference between the value of the ordinate of the Transition Point for the standard data (left) as compared with that derived with the Lockwood data.

synchronous with a relative minimum in the secular oscillation. This indicates that Grand Minima and the Dalton-type minima are due to different phenomena in the solar dynamo system.

The semi-secular and bi-decadal oscillations are nearly the same for the standard and the Lockwood time series but a noticeable difference does exist between the respective secular oscillations during the contemporaneous Grand Maximum, in such a way that, while for the Lockwood homogenized time series the Gleissberg cycles in $a a_{\min }$ and in $R_{\max }$ have the same lengths and are all in phase during the Grand Maximum episode (left-hand panel in Fig. 6 in Duhau \& De Jager 2010), the same does not happen when we use the standard time series (Fig. 1 in De Jager \& Duhau 2009). As a consequence of this difference it appears that, while the path of the long-term variation for the case of Lockwood time series (Fig. 5, right-hand panel) returns to the Transition Point in 2008, the same does not happen when using the standard time series (Fig. 5, lefthand panel). Therefore, in spite of the apparently small qualitative differences (cf. Figs. 2 and 3) between the two time series, a substantial quantitative difference arises regarding the longterm prediction of solar activity. This is so because, while the

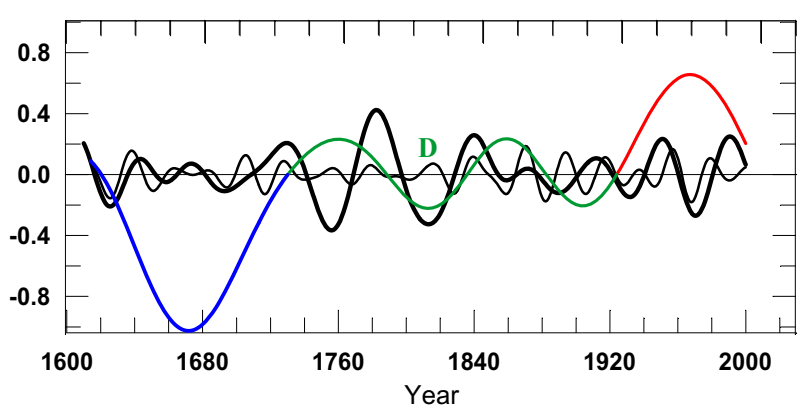

Fig. 6. The three components in which the long-term variations in $R_{\max }$ and $a a_{\min }$ can be decomposed are shown here for $R_{\max }$. These are: the secular cycle (the line with the same sequence of colours as in Fig. 4), the semi-secular and the bi-decadal oscillations (thick and thin lines, respectively).

path in the right-hand diagram indicates that around 2008 a Grand Minimum episode might start, the left-hand diagram indicates that the new episode will be of the Regular type, and so only a Dalton-type Minimum might then develop. 
C. De Jager and S. Duhau: Sudden transitions and grand variations
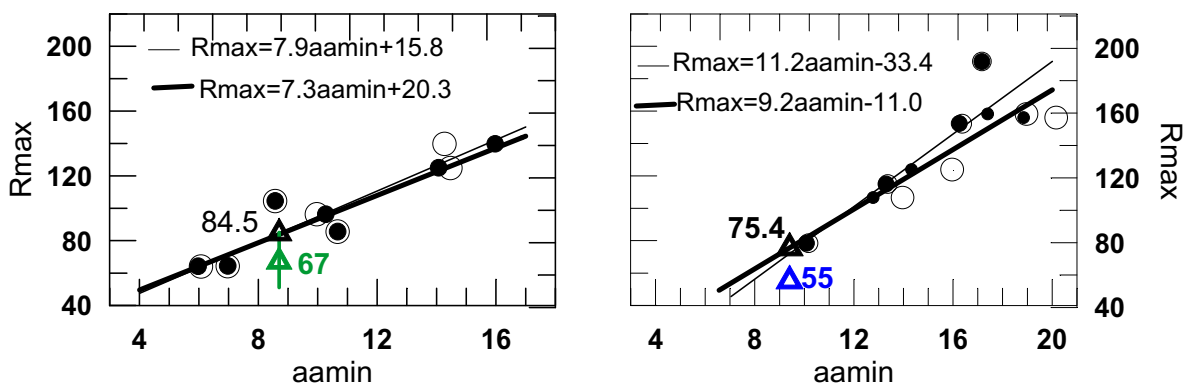

Fig. 7. Sunspot maximum data, $R_{\max }$ vs. geomagnetic index data $a a_{\min }$. The latter have been taken from the standard time series (open circles, thin regression line) and the Lockwood time series (filled circles, thick regression line). The left- and right-hand panels are for the Regular Episode (sunspot cycles \#9 to \#25) and for the Grand Maximum (sunspot cycles \#16 to \#23), respectively. The abscissa values of the green (left) and the red (right) stars are our predictions from the standard $a a_{\min }$ data (De Jager \& Duhau 2009) and for the Lockwood data (Duhau \& De Jager 2010), respectively. The ordinate values are those of the corresponding open triangles in Figure 3. The error bar is included in the lefthand diagram.

Apparently subtle differences appear to be fundamental in forecasting future solar activity. We return to this item in Sections 4 and 5 .

\section{Short-term prediction of solar activity: the present sunspot cycle \#24}

The item that will be discussed here is that of the character of the presently ongoing sunspot cycle \#24. Earlier, we forecasted a maximum sunspot number for Schwabe cycle \#24 of $68 \pm 17$ (De Jager \& Duhau 2009) or of about 55 (Duhau \& De Jager 2010) on basis of the standard or the Lockwood aa data, respectively. As at present the value of $a a_{\text {min }}$ for cycle \#24 is now known from observations we may use the nonlinear relation between the preceding $a a_{\min }$ value and the subsequent $R_{\max }$ value in order to improve our prediction.

The slopes of the regression lines in Figure 7 are mainly determined by the relationship between the respective secular oscillations. That relation is nearly linear but the slope changes strongly after each transition to another grand episode. The amplitudes and lengths of the secular oscillations were comparable during the past Grand Maximum and the Maunder Grand Minimum.

The dispersion of the points around the linear regression lines is due to the nonlinear relationship that exists between the decadal and the semi-secular oscillations. Points deviate most from the linear line when the semi-secular oscillations are strongest, as it happened during sunspot cycle \#19 (the uppermost point in the right-hand panel). This maximum occurred at 1951, when the semi-secular oscillation (Fig. 6) was positive. Therefore, the relevant point in Figure 7 falls above the regression line. Contrary to that is the situation with regard to sunspot cycle \#24. At the moment of its maximum the semi-secular oscillation will be negative. That explains why the predicted values (green and blue triangles) fall below the respective regression lines.

It appears from Figure 7 and the foregoing discussion that a weaker (resp. stronger) sunspot cycle \#24 might be the signature of the start of a Grand Episode, or a Regular one, respectively. However, the variability of the relative phases and strengths of the decadal and semi-secular oscillations at the start of the forthcoming Grand Episode is still unknown, which makes it difficult to evaluate the precise value of the maximum for cycle \#24. But the following consideration will help. Any point will fall close to the regression line when the semi-secular and the bi-decadal oscillations are both small. Therefore, the black triangles in Figure 7 are expected to be close to the actual value of sunspot cycle \#24 if the two shorter oscillations would be of small amplitude. We estimate by extrapolation (see Fig. 6) that both, the bi-decadal and the semi-secular oscillations will be negative during sunspot maximum \#24 and that the respective amplitudes will be comprised between 0 and -5 and between -10 and -20 sunspot numbers. Hence, sunspot maximum \#24 will not be larger than 74 and not smaller than 50, in case we would be dealing with a Regular episode or a Grand Minimum, respectively. This leads to a predicted value of sunspot maximum for cycle \#24 of $62 \pm 12$ sunspot numbers.

To illustrate this last mentioned fact we have plotted (Fig. 8) two pairs of consecutive sunspot cycles that appear to be similar to the pair \#\#23-24. One of them (upper panel) corresponds to the pair that occurred around the period of transition to the Maunder Minimum and the other (bottom panel) is the pair \#\#11-12. That pair belongs to the Regular Episode that lasted from 1724 to 1924 . For comparison, the strengths of the second cycles of each pair are comparable to the strength expected for sunspot cycle \#24 (the square in Fig. 8), but the observed values for the first years of the ascending branch of sunspot cycle \#24 are deviating.

Summarizing: Sunspot cycle \#24 will be the first cycle with a maximum sunspot number below the Transition Point level value since sunspot cycle \#16. The expected maximum sunspot number will be $R_{\max }=62 \pm 12$. It will also be the first cycle of a new Grand Episode, either a Grand Minimum, like the Maunder one, or else a Regular episode, like the 1724-1924 one. We do not know with sufficient precision the value of $a a_{\text {min }}$ $\left(D M_{\max }\right)$ for the last century to be able to forecast, from the path in the phase diagram, which of these two possible kinds of episodes might develop. We return to this problem in the next section.

\section{Significance of the Hallstatt periodicity for determining the character of the forthcoming Grand Episode}

With the present indications that solar activity is moving into another Grand Episode, the question arises what kind of episode that will be. To that end we fist consider the past millennium. It is characterized by a succession of several Grand Minima: viz. the Oort, Wolf, Spörer and Maunder Minima. Steinhilber et al. (2010), who discussed solar activity during 

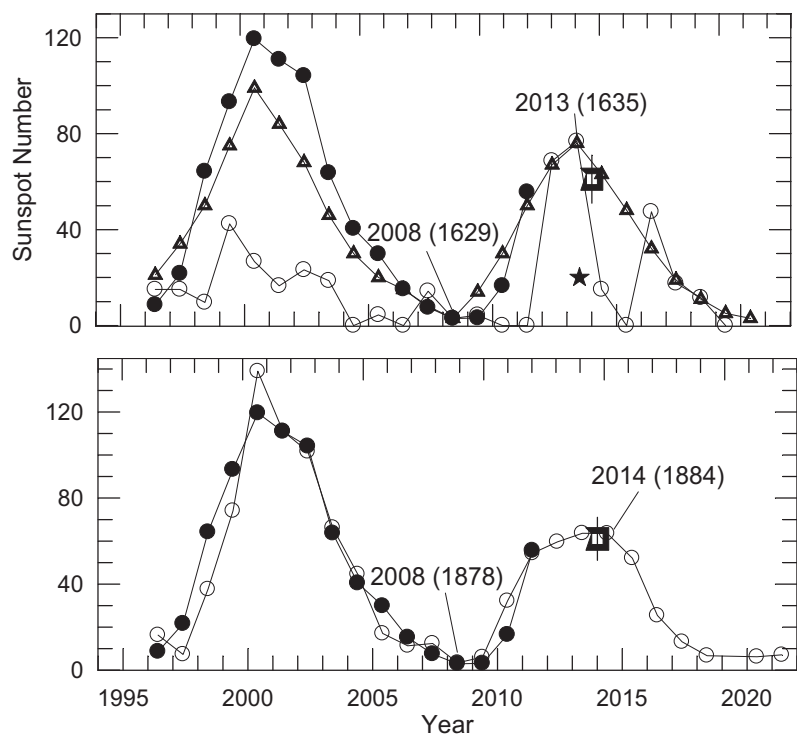

Fig. 8. The open square is our improved prediction of sunspot maximum for cycle $\# 24$, viz. $62 \pm 12$ sunspot numbers, with its estimated error (vertical line). The filled and open circles are taken from the data of Figure 4, but they have been shifted ahead by 378 and 130 years for the upper and the bottom panels, respectively. In the upper panel we have added Nagovitsyn's (2006) annual sunspot number time series (taken from http://www.gao.spb.ru/database/ esai/)(triangles), shifted ahead by 378 years and Vaquero et al. (2010) data (black star). In 1629 and in 2008 (upper panel) there was a transition to another Grand Episode, viz. to the Maunder Minimum and to the forthcoming Grand Episode, respectively. In contrast, the year 1878 (bottom panel) was within the 1724-1924 Regular Episode.

the past 9300 years, concluded that Grand Minima have only occurred in groups during the minimum periods of the Hallstatt oscillation. This cycle has a period of 2300 years (Cliverd et al. 2003), while Steibhilber et al. adopt 2250 years. The latter mention the years $-5300,-3400,-1100$ and $+1500 \mathrm{AD}$ for the minima during which groups of Grand Minima occurred. We illustrate this in Figure 9, which shows a superposition of three recent determinations of the Hallstatt cycle. We observe that the recent series of Grand Minima (Oort to Maunder) occurred during the negative phase of the Hallstatt oscillation, which is in line with the conclusion of Steinhilber et al. (2010).

Crossings of the zero line occurred around the years 919 and 1935. After having passed the zero line, the extreme Grand Maximum of the 20th century occurred. The immanently arising question is what will happen after 2008, when we expect another Grand Episode to start.

For identifying the character of the next Episode we consider in more detail the minima in solar activity during the past minimum branch of the Hallstatt cycle; cf. Figure 10. The date of the transition to the Oort Grand Minimum (vertical blue line in Fig. 10) was determined in a similar way as those of the other three transitions (vertical red lines). The years of the various Dalton-type minima (red letters D) were determined as the years of a succession of at least three maxima that were situated below the transition level.

A comparison of the Schove (1955) and Usoskin et al. (2003) time series shows that they are qualitatively similar. The main difference is that the Hallstatt oscillation is more strongly visible in the Usoskin et al. data. This observation gives confidence in the Schove time series for earlier periods

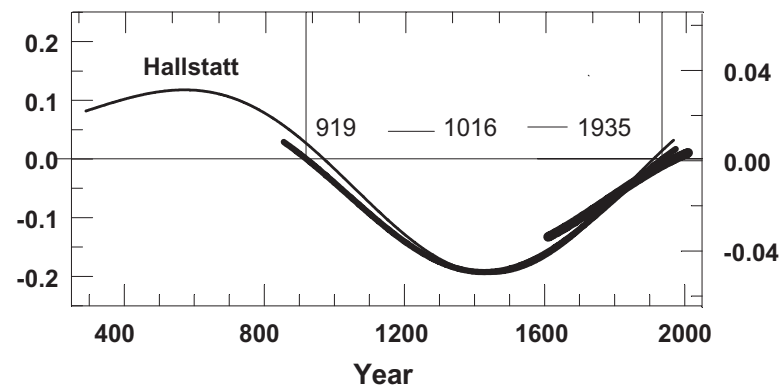

Fig. 9. The Hallstatt oscillation. We show three curves: from thickest to thinner: the international; sunspot number, the Schove $(1955,1961)$ and Usoskin et al.'s (2003) time series, all scaled to fit. The right-hand abscissa corresponds with the historical and Schove time series and the left-hand abscissa is that of Usoskin et al. (2003).

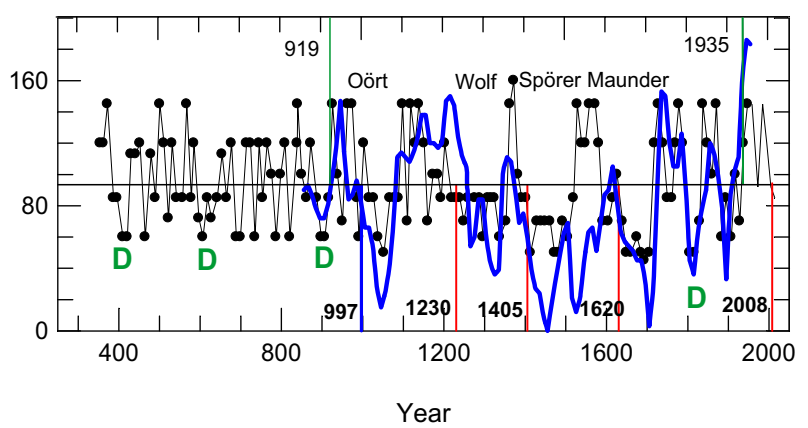

Fig. 10. The data series from Usoskin et al. (2003) (blue line) and Schove (1955) (points). The dashed line is the prediction of Schove for sunspot maxima \#19 to \#24. The vertical red lines indicate the transitions to Grand Minima, as determined by us (reviewed in De Jager \& Duhau (2010) from the Nagovitsyn's (2007) time series. The blue vertical line marks the transition to the Oort Grand Minimum. It was determined here in a similar way as the other transitions (vertical red lines). Letters D mark short (Dalton-type) minima. The black horizontal line is the Transition Point level and the two green vertical lines labelled 919 and 1935 mark the years in which the Hallstatt cycle passes through zero (cf. Fig. 9).

and it also indicates that the Dalton-type minima are not an artefact. A comparison of Figures 9 and 10 confirms that during the period of investigation Grand Minima have exclusively occurred during the negative phase of the Hallstatt oscillation, a conclusion that agrees with that of Steinhilber et al. (2010). This conclusion does not apply to the Dalton-type minima.

It is this observation that leads us to conclude that the next Grand Episode that started in 2008 will most probably be an episode of Regular Oscillations and not a Grand Minimum. However, as shown in this section, we will not be able to safely confirm this prediction as long as the value of sunspot maximum \#24 is not precisely known.

\section{Summary and conclusions}

In previous papers we have forecasted that a new grand solar dynamo episode will start with sunspot cycle \#24. Its character would either be that of a Grand Minimum or that of Regular Oscillations. On the base of the nonlinear relationship that we found between the sunspot numbers at maxima, $R_{\max }$, and the geomagnetic index $a a$ at sunspot minima $a a_{\text {min }}$, as proxies 
for the toroidal and the dipolar field strength, respectively, we predicted that the date of occurrence of the forthcoming sunspot maximum \#24 will be 2013.5. The corresponding $R_{\max }$ value would be $67 \pm 17$ (De Jager \& Duhau 2009) or $\sim 55$ (Duhau $\&$ De Jager 2010). Which of these two values is the more likely one depends on the kind of expected episode. That could either be an episode of Regular Oscillations or else a Grand Minimum. These Grand Episodes are expected to have had their starts after the 2008 phase transition. The decision between these two possibilities depends on two criteria: the observed value of $a a_{\mathrm{min}}$ for polar cycle \#24 in the year 2008.5 and the properties of the three components, in which sunspot maxima time series can be split. These three components are defined as the secular, the semi-secular and the bi-decadal oscillations. In that line we could improve our prediction. We now find $R_{\max }=62 \pm 12$. The choice between the lower and upper bounds of this prediction depends on the property of the forthcoming Grand Episode. The lower value is more probable in the case that we expect a Grand Minimum, while a value close to the upper bound is more probable in the case of Regular Oscillations.

In order to decide between these two possibilities we used the magnetic field phase diagram which is a plot of $R_{\max }$ against $a a_{\mathrm{min}}$. An essential point in that diagram is the Transition Point. Phase transitions, i.e. transitions between Grand Episodes, occur when the $R_{\max }-a a_{\min }$ line hits or passes closely along the Transition Point, when returning to this point after half a secular oscillation. The kind of episode that develops after each phase transition depends on how close to the Transition Point the passing takes place (review in De Jager \& Duhau 2010). Unfortunately we find that the geomagnetic index $a a$ is not known with sufficient precision to lead to a unique answer. In order to solve this dilemma we considered the proxy data for the sunspot maxima time series for the last 1700 years (Schove 1955; Usoskin et al. 2003) and thus we found that the kind of episode that occurs depends on the sign of the bi-millennial Hallstatt oscillation. In line with Steinhilber et al. (2010) we find that Grand Maxima and Grand Minima occur only during the negative phase of this oscillation. The last such phase was between the years 919 and 1935. Prior to 919, when the Hallstatt oscillation was positive, only Dalton-type minima were observed. As we are now, since 1935, in a positive phase of the Hallstatt oscillation, we may not expect a Grand Minimum, certainly not a Maunder-type one, to occur during the ongoing millennium, because it will cover the positive Hallstatt phase. This conclusion is taken in spite of the fact that the insufficient precision of the $a a_{\text {min }}$ data $( \pm 12)$ does not allow for a straight decision between the two possible Grand Episodes. That would only be possible during cycle \#25.

These conclusions, as well as our forecasts (maximum $R$ value for cycle \#24; the character of the forthcoming Grand Episode), do not depend on the exact choice of the year (1924) in which we place the start of the past Grand Maximum - e.g., they will remain the same when the start of the 20th century Grand Maximum would have been chosen one Schwabe cycle later or earlier. On the other hand, the observed peculiar behaviour of the poloidal cycle \#24 confirms that the present transition between Grand Episodes is actually occurring during polar cycle \#24, that is, the polar cycle that maximized between sunspot cycles \#23 and \#24 (cf. Fig. 1). Hence, the dynamo system will be settled in its new regime during sunspot maximum $\# 24$.
Acknowledgements. We are obliged to the two unknown referees for their constructive and very useful remarks

\section{References}

Charbonneau, P., J. Christensen-Dalsgaard, R. Hennig, R.M. Larden, J. Schou, M.J. Thompson, and S. Tomczyk, Helioseismic constraints on the structure of the solar tachocline, Astrophys. J., 527, 445, 1999.

Charbonneau, P., Dynamo models of the solar cycle, Living Rev. Sol. Phys., 7, Available at: http://solarphysics.livingreviews.org/ Articles/lrsp-2010-3, 1, 2010.

Basy, S., and J. Schou, Does the tachocline shows solar cycle related changes? Sol. Phys., 192, 481, 2000.

Brun, A.S., H.M. Antia, S.M. Chitre, and J.P. Zahn, Seismic tests for solar models with tachocline mixing, $A \& A, \mathbf{3 9 1}, 725,2002$.

Cally, D.S., M. Dikpati, and P.A. Gilman, Clamshell and tipping instabilities in a two-dimensional magnetic hydrodynamic tachocline, Astrophys. J., 582, 1196, 2003.

Cliverd, M.A., C. Clarke, H. Rishbeth, T.G.D. Clark, and T. Ulich, Solar activity levels in. Astron 2100, Astron. Geophys., 44, 5.20, 2003.

Duhau, S, and Y. Ch. Chen, The sudden increase of solar and geomagnetic activity after 1923 as a manifestation of a non-linear solar dynamo, Geophys. Res. Lett., 29 (NO. 0), DOI: 10.1029/2001GL013953, 2002.

Duhau, S, and C. De Jager, The solar dynamo and it phase transitions during the last millennium, Solar Phys, 250, 1, 2008.

Duhau, S., and De Jager, The forthcoming Grand Minimum of solar activity, J. Cosmol., 8, 1983, 2010.

De Jager, C., Solar forcing of climate. I Solar variability, Space Sci. Rev., 120, 197, 2005.

De Jager, C., and S. Duhau, Forecasting the parameters of sunspot cycle 24 and beyond, J. Atmos. Sol. Terr. Phys., 71, 239, 2009.

De Jager, C., and S. Duhau, The variable solar dynamo and the forecast of solar activity; effects on terrestrial surface temperature, in J.M., Cossia, ed., Proceedings of the global warming in the 21th century. NOVA science publishers, Hauppauge, NY, pp. 77-106, 2011.

De Jager, C., S. Duhau, and B. van Geel, Quantifying and specifying the solar influence on terrestrial temperatures, J. Atmos. Sol. Terr. Phys., 72, 526, 2010.

Fisher, C.H., Y. Fan, B.W. Longcope, H.G. Linton, and A.A. Pevtsov, The solar dynamo and emerging flux - (invited review), Sol. Phys., 192, 119, 2000.

Hathaway, D.H., R.M. Wilson, and E.J. Reichmann, A synthesis of solar cycle predictions, J. Geophys. Res., 104, 22388, 1999.

Hujeirat, A., and H.W. Yorke, On the MHD structure of the solar tachocline. Steady and dynamical solutions, New Astron., 3, 671, 1998.

Kùker, M., G. Ruduger, and M. Schultz, Circulation dominated solar shell dynamo with positive alpha effect, $A \& A, \mathbf{3 7 4}, 301,2001$.

Legrand, J.P., and P.A. Simon, A two component solar cycle, Sol. Phys., 121, 187, 1991.

Layden, A. C., P. A. Fox, J. M. Howard, K. H. Dsarajedini, and S. Sofia, Dynamo-based scheme for forecasting the magnitude of solar activity cycles, Sol. Phys., 132, 140, 1991.

Lockwood, M., D. Whiter, B. Hancock, R. Henwood, T. Ulich, H.J. Linthe, E Clarke, and M.A. Clilverd, The long-term drift in geomagnetic activity: calibration of the $a a$ index using data from a variety of magnetometer station, Available at: http://www.eiscat.rl.ac.uk/Members/mike/publications/pdfs/sub/241_Lockwood_ aa correct S1a.pdf, 2006.

Mayaud, P.N., Analysis of storm sudden commencements for the years 1868-1967, J. Geophys. Res., 80, 111, DOI: 10.1029/JA080i001p00111, 1975.

Nagovitsyn, Y., To the description of long-term variations in the solar magnetic flux: the sunspot area index, Astron. Lett., 31 (8), 
557, Translated from Pis'ma v Astronomicheskiı Zhurnal, 31 (8), 622, 2005.

Nagovitsyn, Yu. A., Solar and geomagnetic activity on a long time scale: reconstructions and possibilities for predictions, Astron. Lett., 32 (5), 344, Translated from Pis'ma v Astronomicheski1 Zhurnal, 32 (5), 382, 2006.

Nevanlinna, H., and E. Kataja, An extension of the geomagnetic index series aa for two solar cycles (1844-1868), Geophys. Res. Lett., 20, 2703, 1993.

Ohl, A.I., Forecast of sunspot maximum number of cycle 20, Solnice Danie, 9, 84, 1966.

Ossendrijver, M., Understanding the solar dynamo, Astron. Astrophys. Rev., 11, 287, 2003.

Russell, C.T., On the possibility of delivering interplanetary and solar parameters from geomagnetic records, Sol. Phys., 42, 259, 1975.

Russell, C.T., and T. Mulligan, The 22-year variation of geomagnetic activity: Implications for the polar magnetic field of the sun, Geophys. Res. Lett., 22, 328, 1955.

Schatten, K.H., P.H. Scherrer, L. Svalgaard, J.M. Wilcox, Using dynamo theory to predict the sunspot number during solar cycle 21, Geophys. Res. Lett., 5, 411, 1978.

Schove, D.J., The sunspot cycle, 649 B.C. to A.D. 2000, J. Geophys. Res., 60, 127-156, 1955.
Schove, J.D., Solar cycles at the spectrum of time since 200 B.C., Ann. New York Acad. Sci., 95, 107, 1961.

Solanki, S.K., B. Inhester, and M. Schüssler, The solar magnetic field, Rep. Prog. Phys., 69, 563, DOI: 10.1088/0034-4885/69/3/ R02, 2006.

Steinhilber, F., J.A. Abreu, J. Beer, and K.G. McKracken, Interplanetary magnetic fields during the past 9300 years inferred from cosmogenic radionuclides, J. Geophys. Res., 115, 111, DOI: 10.1029/2009JA014193, 2010.

Svalgaard, L, E. W. Cliver, and P. Le Sager, IHV, a new geomagnetic index, Adv. Space Sci., 34, 436-439, 2004.

Tobias, S.M., The solar dynamo, Philos. Trans. R. Soc. London, Ser $A, \mathbf{3 6 0}, 2741$, DOI: 10.1098/rsta.2002.1090, 2002.

Usoskin, I.G., S. Solanki, M. Schüssler, K. Mursula., and K. Alanko, Millennium-scale sunspot number reconstruction; evidence for an unusually active sun since the 1940s, Phys. Rev. Lett., 91 (21), 211101-1, 2003

Vaquero, J.M., M.C. Gallego, J.G. Usoskin, and G.A. Kovaltsov, Revisited sunspot data: a new scenario for the onset of the Maunder Minimum, Astrophys. J., 713, L24, 2010.

Vennerstroem, S., Long-term rise in geomagnetic activity-A close connection between quiet days and storms, Geophys. Res. Lett., 27, 69, 2000. 